\title{
SIMULATING THE AM PRODUCTION FACILITY: A CONFIGURABLE SOFTWARE TOOL FOR STRATEGIC FACILITY-LEVEL PLANNING
}

\author{
Eldar Shakirov \\ Center for Design, Manufacturing and Materials, \\ Skolkovo Institute of Science and Technology, \\ Moscow, Russia \\ Clement Fortin \\ Space Center, \\ Skolkovo Institute of Science and Technology, \\ Moscow, Russia
}

\author{
Kaitlyn Gee, Haden Quinlan, A. John Hart ${ }^{1}$ \\ Department of Mechanical Engineering, \\ Massachusetts Institute of Technology, \\ Cambridge, MA, USA \\ Ighor Uzhinsky \\ Center for Design, Manufacturing and Materials, \\ Skolkovo Institute of Science and Technology, \\ Moscow, Russia
}

$\begin{array}{ll}\text { SLM } & \text { Selective Laser Melting } \\ \text { ABC } & \text { Activity-Based Costing } \\ \text { DES } & \text { Discrete Event Simulation }\end{array}$

\section{INTRODUCTION}

Metal Additive Manufacturing (AM) technologies are of growing industrial interest in the wake of recently announced production applications. These applications include the production of functional, end-use components for aerospace, medical, and selected automotive applications in significant production quantities (10,000's and greater) [1-3]. This is especially true for laser powder bed fusion processes for metals, which are commonly referred to as "Selective Laser Melting" (SLM) or "Direct Metal Laser Sintering” (DMLS) [4, 5]. Today, AM's production economics often limit its use to applications that derive significant value from component performance and/or weight savings. However, the increasing maturation of the AM technology landscape will enable greater cost-efficiencies for larger product volumes, justified via not only part performance but also by system-level dynamics that yield systemic efficiencies in supply-chain response, assembly minimization, or other advantages [6]. Therefore, it is essential that organizations are able to explore different implementation scenarios projected against the current and future capabilities of AM production systems in order to define efficient deployment strategies.

To understand the economic implications of deploying AM at different production scales, in different application contexts,

\footnotetext{
${ }^{1}$ Contact author: ajhart@mit.edu
} 
and with variable levels of overall machine performance and efficiency, the recurrent and non-recurrent costs of production must be investigated scrupulously [7]. Management literature suggests numerous methods to account for and assign production costs. For the model proposed in this work, a time driven Activity-Based Costing (ABC) method is utilized for its intuitive and configurable mathematics, and its ability to be granularized to accurately account for a wide range of production steps [8]. Described succinctly, an ABC model will define discrete production activities (which can be as granular as an operator transporting a component between workstations, or as general as an entire print or build cycle). Each activity is assigned a cost and a time, and these values are divisible by the number of units worked on (or the overall throughput of the system) to produce a cost per part which can be linked intrinsically to the constituent production tasks from which the final component cost is derived.

Consequently, the output fidelity of an $\mathrm{ABC}$ model directly depends on the accuracy of time and cost estimates for each of the constituent activities accounted for within the model. With this in mind, this research utilizes simulation to capture and parameterize individual production tasks which drive final component cost. For this simulation, the Discrete Event Simulation (DES) method, which is one of the most widely used method in manufacturing and business, has been chosen for its good applicability for the tasks in scheduling, production planning, tactical and operational decision-making, and others [9]. Further discussion of the DES method is described in Section 2 of this paper.

Therefore, this work aims to develop a simulation-based cost model, which can be used as an instrument for comprehensive techno-economic analysis of a proposed configuration of an AM production facility which utilizes SLM equipment for component printing. Certain characteristics of the AM production process (e.g. staffing levels, shift patterns, or build time) have been investigated previously [10]. However, past work is limited in scope, and has not considered the cost and schedule implications of post-processing activities. Postprocessing is a catch-all term used to describe secondary activities performed after the printing step, ranging from thermally relieving printed components to surface finishing those components using different methods. In the SLM process specifically, a stress relieving heat treatment cycle is required for each component (which may be batched into a single furnace cycle). In addition, SLM components typically require a large amount of sacrificial support geometry, as is shown on the suspension bell-crank (Fig. 1, left); however, some SLM components do not (Fig. 1, right). Support geometries are comprised of the same material as the printed component, and therefore require a manually intensive removal process. Finally, other finishing steps may be taken to improve the part's surface properties (e.g. sanding, blasting, tumbling) or its mechanical and metallurgical properties (e.g. hot-isostatic pressing). The

2 Original picture source: https://www.linkedin.com/pulse/topologicaloptimisation-really-optimal-marc-saunders

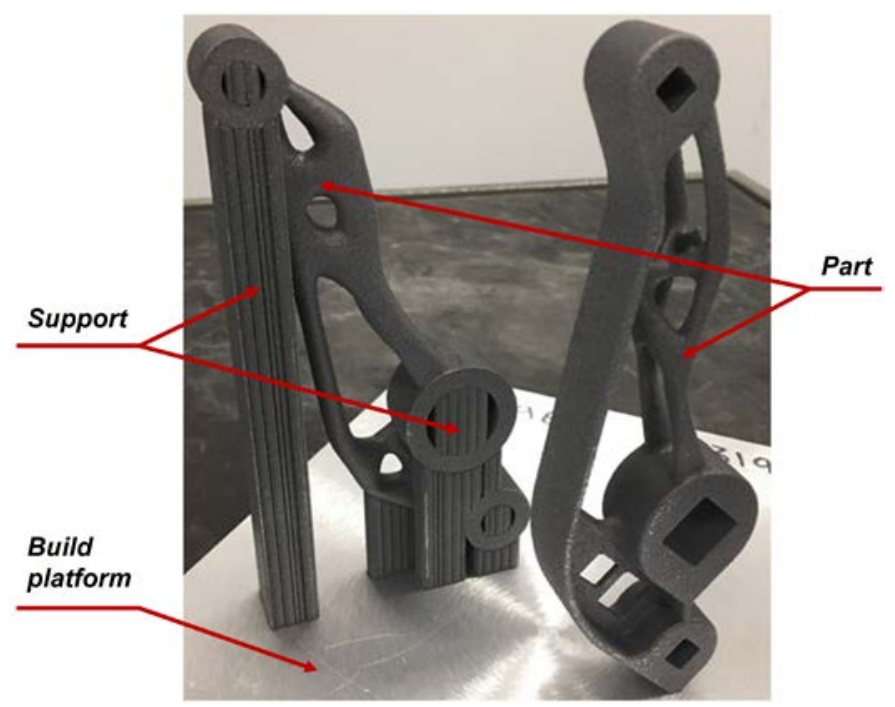

FIGURE 1: TWO SUSPENSION BELL-CRANKS PRODUCED VIA SLM. LEFT: GEOMETRY-OPTIMIZED BELL-CRANK WITHOUT MANUFACTURING CONSTRAINTS APPLIED. RIGHT: GEOMETRY-OPTIMIZED BELL-CRANK WITH MANUFACTURING CONSTRAINTS APPLIED ${ }^{2}$

cost and duration of post-processing tasks are a necessary inclusion in any robust cost model of the SLM process, and an important contribution of this work to the field.

Taken in total, this study asks, "For components manufactured via an SLM-enabled production facility, how can we identify their main cost and lead time drivers by means of simulation modeling? Can this modelling approach provide a methodology for decisionmakers to derive the optimal configuration $^{3}$ of an AM production facility for a pre-defined geometry and quantity of parts?"

\section{MATERIALS AND METHODS}

To provide a simulation approach with a resolution commensurate to that of $\mathrm{ABC}$, this research employs the DES technique. For this, the SLM production workflow is segregated into a necessary set of states, where the transition from one state to another occurs instantaneously upon the conclusion of previous events. For example, consider the processing unit, an AM machine, which after completing a "cooldown" event switches into a "waiting" state, i.e. holds until the operator would execute a "coming over" event and turn the machine into the "service" state. By using a high-degree of detail in characterizing events, the DES method produces a granular view into production activities.

The model has been implemented in Tecnomatix Plant Simulation software, which makes use of integrated features such as: (a) object-oriented programming, (b) multi-level experimental design, (c) and data exchange with external software. Feature (a) serves as an instrument for determination

\footnotetext{
${ }^{3}$ For the intents of this study, configuration refers to both physical configuration and selection of equipment, as well as number of plant employees and standard operating procedures such as packing strategy.
} 


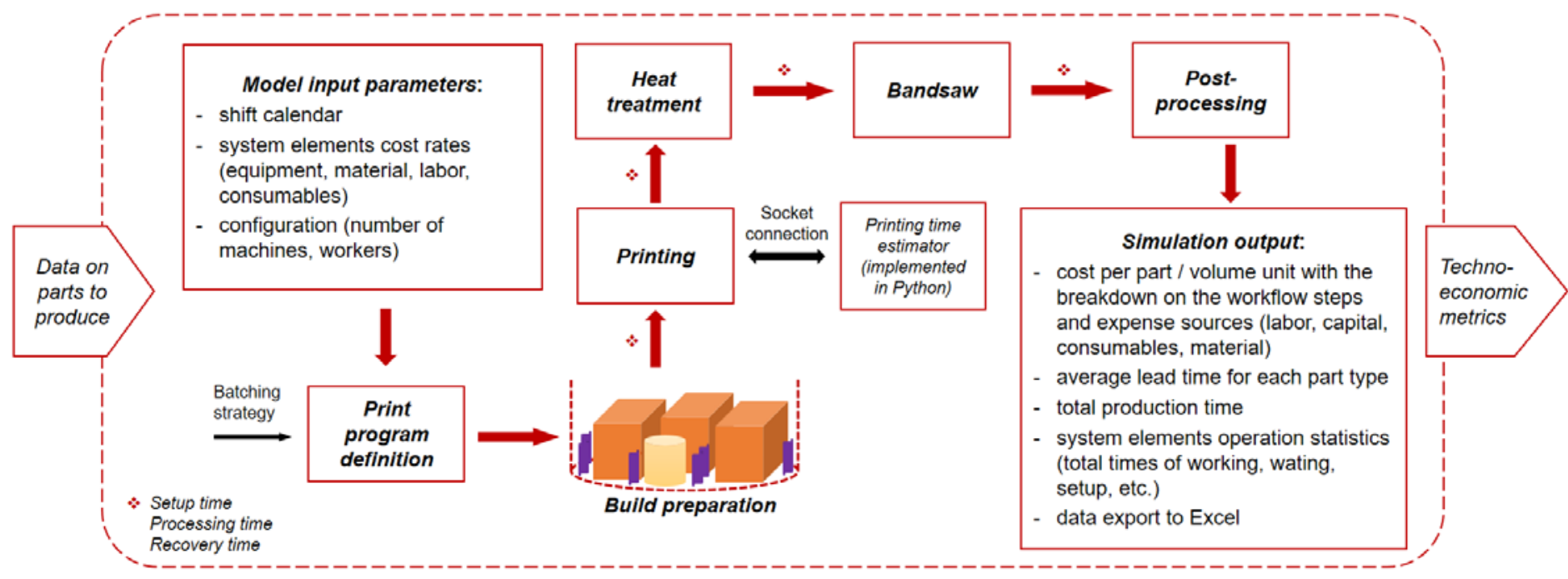

FIGURE 2: MODEL OVERVIEW

of the accurate behavioral logic of the objects modelled (e.g. machines, workpieces, workers) and accounts for their associated costs; whereas the latter two features (b, c respectively) play a role in facilitating the analysis of different model configurations by importing the parameters of the simulation and exporting the results. The model covers a generalized SLM workflow, which consists of four primary stages: (1) build preparation, (2) printing, (3) heat treatment, and (4) post-processing; the model is described in full in Figure 2. The following subsections describe the logic of the model in detail.

\subsection{Input of parts data}

The setup of the model begins with the definition of simulation parameters. The set of parts to be produced is defined in an Excel spreadsheet providing information necessary to determine the processing parameters for each part (Table 1), approximating each part's geometry by its bounding box dimensions and fill density. The other general settings, such as shift calendar, cost of machines, material, and labor are defined in the model. The default shift pattern is two-shift, each of nine hours, and it is assumed that all parts are produced from the same material within a given production run.

\subsection{Batching the parts into different builds}

Based on the imported data, the model determines how parts will be queued and allocated to different equipment based on a batching algorithm. The batching approach is chosen by the software user. The tool compares three distinct batching strategies: mixed max fill, single, and max fill (Fig. 3). Mixed max fill aims to minimize the per-build costs associated with setting up the printers by assigning the maximum number of parts per build job, which may include components with different geometries and bounding box dimensions. Single attempts to minimize the overall lead time (i.e., the time required for one part to complete all production stages, including non-working hours)
TABLE 1: PART INPUT DATA

\begin{tabular}{|l|l|l|}
\hline Parameter & Purpose & Range \\
\hline Part ID & $\begin{array}{l}\text { To track the part and accumulate } \\
\text { the costs associated with the } \\
\text { same part type }\end{array}$ & $1-9$ \\
\hline Quantity & $\begin{array}{l}\text { The number of each part to be } \\
\text { produced }\end{array}$ & $1-100$ \\
\hline Length, Width & $\begin{array}{l}\text { Area of the bounding box in the } \\
\text { plane parallel to the build plate }\end{array}$ & $\leq 0.06 \mathrm{~m}$ \\
\hline Height & Volume of the bounding box & $\leq 0.08 \mathrm{~m}$ \\
\hline $\begin{array}{l}\text { Part relative } \\
\text { density }\end{array}$ & $\begin{array}{l}\text { To derive a mass of the part as a } \\
\text { fraction of its bounding box }\end{array}$ & $\leq 1$ \\
\hline $\begin{array}{l}\text { Support relative } \\
\text { density }\end{array}$ & $\begin{array}{l}\text { To derive the mass of supports } \\
\text { as a fraction of part mass }\end{array}$ & $\leq 0.99$ \\
\hline $\begin{array}{l}\text { Post- } \\
\text { processing } \\
\text { complexity } \\
\text { factor }\end{array}$ & $\begin{array}{l}\text { A multiplier for the total effort } \\
\text { necessary for post-processing }\end{array}$ & $1-4$ \\
\hline Given days & To prioritize the order of parts & $1-30$ \\
\hline
\end{tabular}

for each part by assigning a single part to each build job. The max fill strategy, which attempts to both reduce the lead time and costs of auxiliary steps, such as machines warm-up and cooldown, batches only parts of the same ID within each build. As with the mixed max fill strategy, the max fill batching approach allocates the maximum number of components per build job as can be produced according to the cross-sectional area of the part's bounding box and the build area of the equipment utilized.
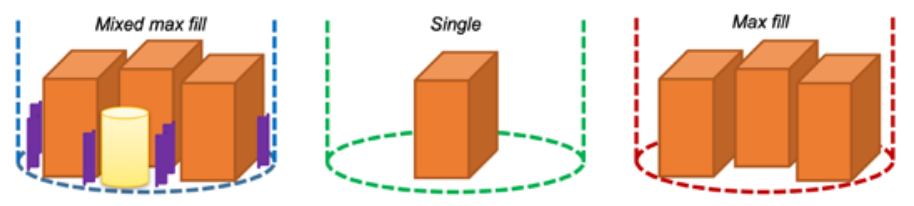

FIGURE 3: BATCHING STRATEGIES 
When the simulation commences, each machine is idle prior to the assignment of parts. As builds are assembled, they are allocated to the first available machine in the system. For the actual batching of components, the model operates sequentially by allocating the maximum number of parts one-by-one, according to the predefined batching strategy, to each build job. It starts first with the highest priority components as determined by the Given Days parameter, before assigning components of lesser priority. When the area left inside of a machine is insufficient to add an additional part to that build job, the model then begins to allocate the rest of the parts into the next available build job or machine until all parts have been assigned. After this, the model distributes the next part (as determined by the part's stated priority) in a queue among the first and subsequent builds for the mixed max fill strategy, and only among the subsequent builds for the max fill strategy. When all parts have been assigned, the model begins the build preparation stage.

\subsection{Build preparation}

The build preparation stage simulates the process of receiving the $3 \mathrm{D}$ digital files of part geometries according to the specification of the next scheduled build for each machine. This specification is derived from the previous batching approach. This model assumes that build preparation takes 15 minutes per part; thus, the total time is a multiple of part quantity in the build. A complementary time selection criterion is based on the history of build preparation: if the upcoming build consists of precisely the same list of parts as in the previously prepared build, then the process represents a retrieval of the previously developed print program and lasts for five minutes total. This therefore assumes that the first build preparation activity is always successful (as subsequent build preparation activities for the same build are presumed to be trivial).

For cost accounting, the build preparation stage involves both non-recurring costs (in the form of expenses on software licenses), and recurring costs spent on AM engineers' effort. The output of this step is then transferred to the printing stage.

\subsection{Printing}

The printing stage simulates the receipt of control instructions (e.g., the toolpath employed by the machine) for the build and defines the time required for different operational tasks performed by the machine during the printing stage. These tasks include machine setup time, processing time, and recovery time; each of these tasks are accompanied by further sub-tasks. The setup time consists of time spent on powder refill, build installation, and machine warm-up; times follow a random normal distribution with mean times of 20, 25, and 30 minutes, respectively. The powder refill with a dispenser duct happens only when there is not enough powder in the refilling module necessary for the volume of an upcoming build's bounding box.

Processing time is derived from the total mass of the build (this includes both parts mass and supports mass) and a constant volumetric build rate expressed in $\mathrm{cc} / \mathrm{hr}$, which is specified by the user. For the purposes of this study, an approximate build rate of $10 \mathrm{cc} / \mathrm{hr}$ (of solid material) is chosen based on data provided by machine manufacturers. Ongoing work seeks to establish a more accurate build time estimation, by prompting external software to provide an accurate time estimation through physicsbased simulation of the SLM printing process for a given geometry and build strategy. So far, this has been realized in the form of the local client-server connection through a two-way socket communication with the Python script in which the time estimation is encoded.

The recovery time of an SLM machine accounts for cool down, build removal, and removal of excess powder with the total mean time of 75 minutes. Also, the SLM machines undergo periodic cleaning and preventative maintenance (PM) according to a user-defined cadence; for the purposes of this work, this PM process happens each week and lasts for three hours. These values are chosen from the best-practice experience of an AM production facility which uses SLM for medical devices.

The logic of the printing machine also accounts for the time available before the end of the final shift in a given day: if there is not enough time to finish the printing process within a single day, then the printing process would pause at the conclusion of the second shift until the commencement of the subsequent shift the following day. However, there is an exception for large and time-consuming builds: if the printing process demands time that exceeds the total available time from subsequent shifts before the night break, if any, then the printing process starts when it was prompted with an assumption of overnight worker involvement. Though it is possible for print cycles to run asynchronously to scheduled worker shift patterns (e.g. to run continuously throughout the night even when there are no employees present to monitor the process), this work assumes a position of overt caution where no printing occurs except when an operator is physically proximate and working.

For cost accounting, the printing stage contributes via the costs of printing feedstock material (e.g. metal powder) and the costs of the printing process itself. Recurring costs include the cost of material spent on the part, its supports, and the waste material that has not been recycled and which cost is spread evenly among the parts in the build. The material consumed by the part and its supports can be easily derived from the parts' specifications, whereas the wasted material is calculated as a fraction of the total unused volume of powder remaining in the build chamber after printing. Some powder waste is inevitable, as in-process effects (e.g. spatter) denature the powder morphology and make it unsuitable for additional printing [10]. For the purposes of this work, a waste fraction of $10 \%$ is used; as with the build preparation parameter, the true value of this fraction is context-dependent and is more accurately derived empirically than theoretically. To define the mass of the total unused powder, the model takes the height of the build, which is equal to the highest part in the build, and subtracts from the mass of a bounding cylinder (or box, depending on the machine) the total mass of the build. The model assumes that the total mass of powder used per build is the mass required to clear the highest part of the build. Therefore, the mass of total unused powder is the total mass of powder minus part and support mass; it is then 
multiplied by the waste fraction to arrive at an amount and cost for wasted material.

The non-recurring costs of printing are cost associated with the machine: (a) its purchase cost, which is affected both by a depreciation term and the cost of capital (both are user-defined parameters); (b) a yearly maintenance cost (assumed here to be $5 \%$ of the machine's retail cost, paid annually); (c) the cost of infrastructure associated with setting up the production facility (assumed here to be 50\% of the machine cost, paid once); (d) and general administrative overhead. From these costs, the model determines an average annual cost of ownership per machine, which is then divisible by the total number of operational hours in a given calendar year (52 weeks, with 5 days per week) to arrive at a machine cost per hour. These costs are then applied proportionally to the time the machine is occupied per the buildtime calculation and set-up and cool-down times specified by the user to arrive at a cost per-build, which is then assigned fractionally to each part according to the part's mass (here, mass is used as a proxy for a part's contribution to the overall build time).

The recurring costs include consumables which scale differently. Gas consumption scales directly with build time, as the model assumes a constant volumetric flow rate (expressed in $\mathrm{L} / \mathrm{hr}$ ) to a machine during its warm-up, printing, and cool-down. Cost is assigned according to the cost per $\mathrm{L}$ of gas. Other consumable costs scale with the number of builds, irrespective of their duration, as some components are replaced either wholly (e.g. filtration units) or fractionally (e.g., the substrate upon which components are printed is surfaced between each build and, after a certain number of builds, is discarded) per-build. Additionally, the cost of labor is accounted for according to variable full-time equivalents (FTEs) for operators' work during print supervision and setup. The total cost of printing the build is then distributed among the parts proportionally to their supportincluded masses.

\subsection{Heat treatment}

After the printing step, the processed build heads to the heat treatment stage for stress relief. Similarly to the printing stage, heat treatment contributes to both non-recurrent and recurrent costs of the part. The processing time assumed to be constant and is equal to two hours for each build, though in future work the heat treatment schedule should be chosen according to the material, and build configuration (i.e., adjusting the heating and cooling schedule if necessary). The cooldown occurs outside the furnace and lasts for one hour. As a result, the costs are distributed in accordance with masses of parts.

\subsection{Post-processing}

The use of a band saw for separating parts from the build platform is assumed; post-processing thus includes the sawing operation, additional manual removal of support material, and other finishing operations. Each of these processes include the costs of equipment, labor, and consumables. Activity durations are derived simply. Sawing time is a constant value (units $\mathrm{cm}^{\wedge} 2 / \mathrm{hr}$ ), which is then proportional to the cross-sectional area of all parts in the build. Support removal time is proportional to the mass of support material for each part (and is further modified by the post-processing complexity factor).

\subsection{Total cost}

The sum of all costs gives the total costs for each part type. In addition, idle time costs are also considered. Idle time is the time that machines, including the printers, the furnace, and bandsaw, spend waiting to be operated or maintained (e.g., machines may wait idle if their operation is contingent upon the execution of a previous production step which has not been completed). When all parts have been produced, based on the resulting statistics of each object in the system, the model calculates the total idle times for each machine and derives the idle time costs by multiplying the times with corresponding machine hourly rates. These costs are then distributed among the part types proportionally to their average lead times (the assumption is that the longer it takes to produce the part, the higher idle times portion would be assigned to it). Taken in total, the model can then elucidate the total cost per part and discretize this cost into either the cost of individual workflow steps, or the values of recurring and non-recurring cost components.

\subsection{Scenario planning using the model}

For the model to be practically useful, it must be able to process a large volume of data rapidly and accurately, and therefore explore a variety of operation scenarios for the AM shop floor. We sought to investigate approximately 2000 scenarios, which are combinations of batching strategy, part sizes, order priorities, equipment count, production volume, and other relevant characteristics. To analyze these variables, the model must possess the following features:

a. All operational times associated with manual work follow a random normal distribution that accounts for natural process time fluctuation.

b. To enable the large-scale run of multi-level experiments via a built-in "Experiment Manager" tool, the model is capable of self-reconstruction based on parametric variation. For each change in any of the parameters, the model needs to run another set of observations to generate a dataset that can be analyzed. There were 20 observations per experiment within a confidence interval of $95 \%$.

c. Integration with Microsoft Excel or other spreadsheet-based software tool for convenient centralized input of parts data (see Table 1) and output of analytics.

Using this approach, the model is capable of studying a necessary set of scenarios and characterizing trade-offs for the purposes of practical analysis.

\section{RESULTS AND DISCUSSION}

The overall goal of this work is to present a preliminary model capable of examining how a set of planning decisions affect the technical and economic performance of an AM production facility. We present a demonstrative analysis that explores the influence of the following four parameters on parts costs and lead times: (a) batching strategy (see Section 2.2), (b) 
the number of printers, (c) the number of furnaces, and (d) the number of operators. The selected reference model of the AM machine is the EOS M100, which includes a cylindrical build chamber $100 \mathrm{~mm}$ in diameter and $95 \mathrm{~mm}$ in height. First, we investigated the characteristics of producing one part type, and then the production of a set of different parts.

\subsection{Producing a single part type}

First, we consider the cost of manufacturing an abstract part with given dimensions and material characteristics (see Table 1). The analysis shows the effect of employing additional AM machines or changing the batching strategy. The line graphs are given in Fig. 4a and 4b demonstrate how cost and lead time scale with production volume, respectively. When comparing batch strategies for manufacturing 100 parts with four SLM machines, we observe that the single batch strategy has a higher associated unit cost (+50\%), and a reduced associated lead time (-26\%). The cost breakdown analysis for 100 parts production in Table 2 proposes three reasons: a higher materials expenditure, driven by a higher volume of powder to be recycled (and wasted); per-build costs are both increased (as a function of more builds being needed to produce an equivalent number of parts), and each perbuild cost is applied entirely upon a single part; and the increase of idle time costs as a result of unoptimized production flow.

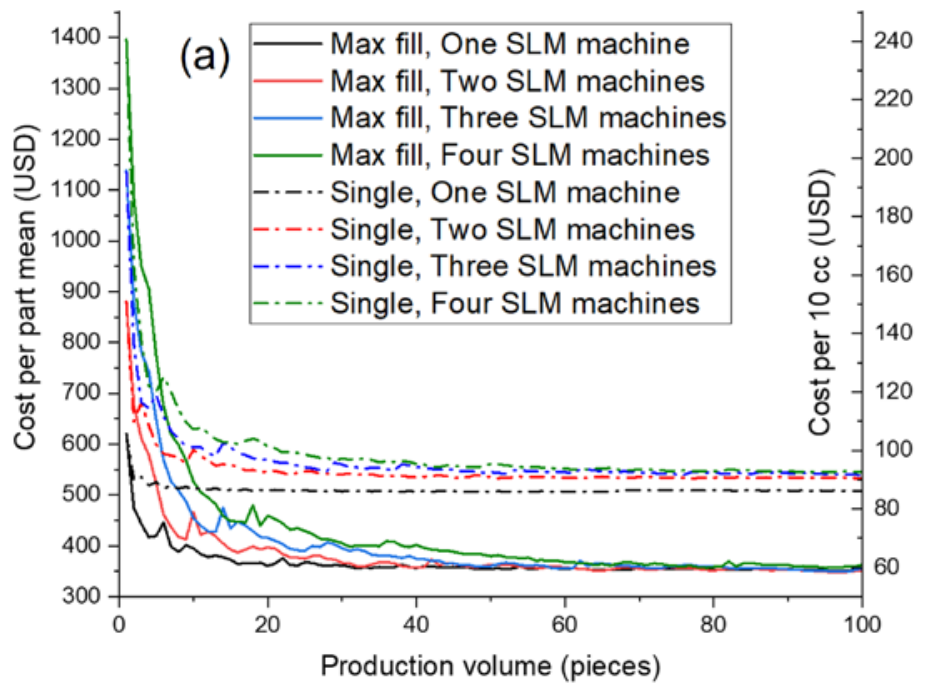

FIGURE 4: (a) COST PER PART AND (b) LEAD TIME VERSUS PRODUCTION VOLUME FOR A SINGLE PART GEOMETRY

\subsection{Producing multiple part types}

The second study simulates a manufacturing process for the production of quantities of parts with different characteristics listed in Table 1. In this scenario, 114 total parts are considered. The component dimensions are comparable to small dental, medical, and jewelry components. Further, these components vary in quantity, post-processing complexity, and given days.

From this experiment, we can observe the influence of the batching strategy on unit costs, individual cost elements breakdown, and component lead times (Fig. 5; values are given for the production system that utilizes only one printer). As in PRODUCTION OF 100 PARTS
TABLE 2: COST PER-PART BREAKDOWN FOR MAX FILL STRATEGY AND SINGLE STRATEGY EMPLOYED IN THE

\begin{tabular}{|l|c|c|c|c|}
\hline \multirow{2}{*}{$\begin{array}{l}\text { Cost } \\
\text { constituent }\end{array}$} & \multicolumn{2}{|c|}{ Max fill strategy } & \multicolumn{2}{c|}{ Single strategy } \\
\cline { 2 - 5 } & Value & Share & Value & Share \\
\hline $\begin{array}{l}\text { Build } \\
\text { preparation }\end{array}$ & $\$ 0.7$ & $0.2 \%$ & $\$ 0.2$ & $0.04 \%$ \\
\hline $\begin{array}{l}\text { Printing: } \\
\text { material }\end{array}$ & $\$ 30.9$ & $8.8 \%$ & $\$ 47.4$ & $9.31 \%$ \\
\hline $\begin{array}{l}\text { Printing: } \\
\text { machine usage }\end{array}$ & $\$ 157.4$ & $44.7 \%$ & $\$ 189.5$ & $37.22 \%$ \\
\hline Printing: labor & $\$ 73.7$ & $20.9 \%$ & $\$ 120.3$ & $23.63 \%$ \\
\hline $\begin{array}{l}\text { Printing: } \\
\text { consumables }\end{array}$ & $\$ 36.5$ & $10.4 \%$ & $\$ 40.3$ & $7.9 \%$ \\
\hline Heat treatment & $\$ 8.8$ & $2.5 \%$ & $\$ 35.9$ & $7.1 \%$ \\
\hline $\begin{array}{l}\text { Post- } \\
\text { processing }\end{array}$ & $\$ 30.8$ & $8.8 \%$ & $\$ 34.1$ & $6.7 \%$ \\
\hline Idle time & $\$ 13$ & $3.7 \%$ & $\$ 41.5$ & $8.1 \%$ \\
\hline Total cost & $\$ 351.8$ & $100 \%$ & $\$ 509.2$ & $100 \%$ \\
\hline
\end{tabular}

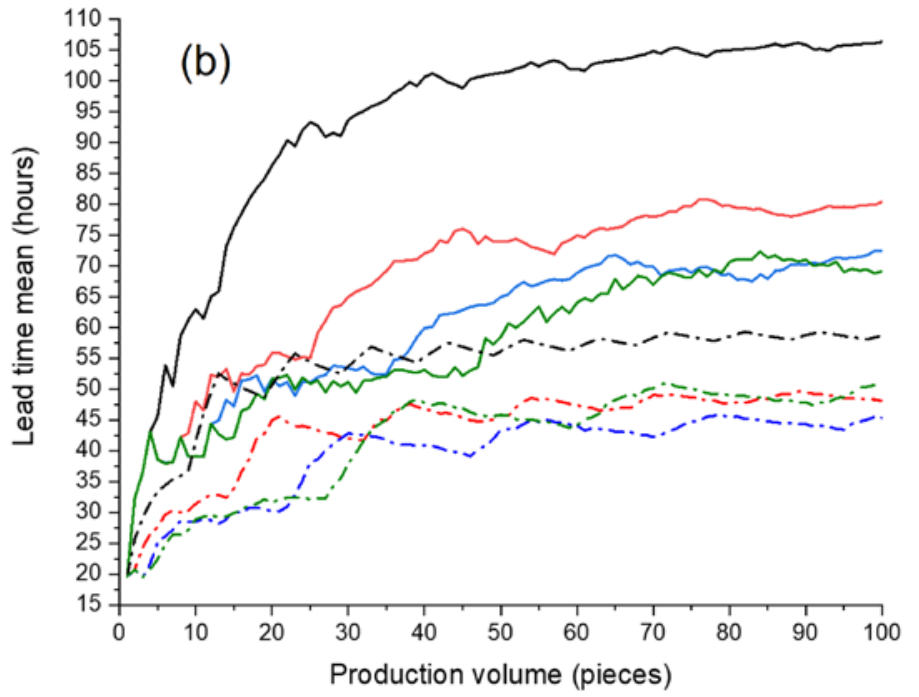

the first study, we observe the cost of a production rate increase when going from the mixed max fill strategy to the single strategy. Moreover, we observe that going from the mixed max fill strategy to the max fill strategy marginally increases the unit cost across most of the part IDs. In contrast, the average lead time significantly reduces.

Going further, as shown in Figure 6, the tool can reveal tradeoffs in average cost metrics (represented as cost per cubic centimeter), average lead time (defined as the quantity-adjusted average of lead time means across part IDs), and the total production time (defined as the time required to produce all parts within the study). Moreover, the experiments reveal that 


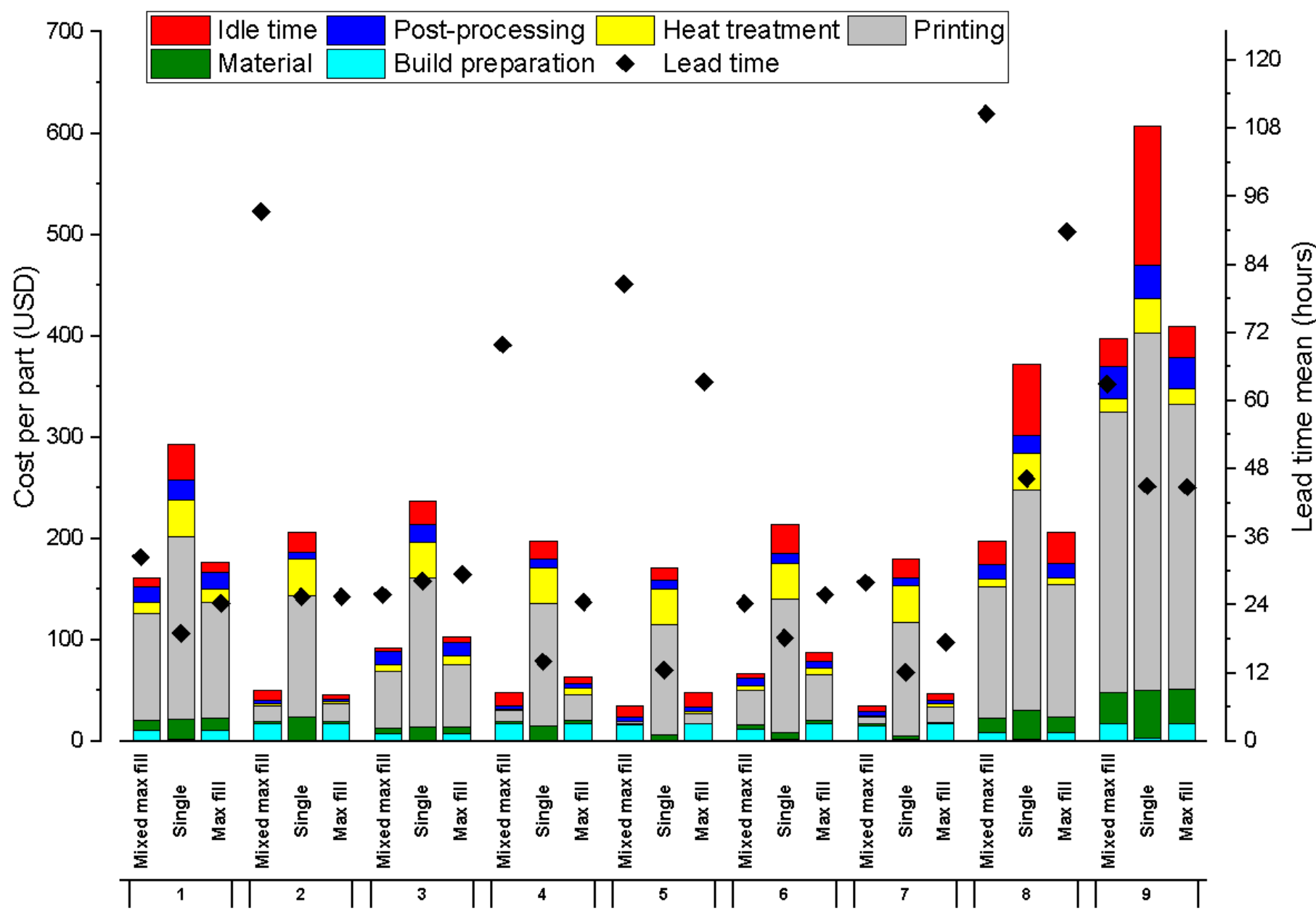

FIGURE 5: SUMMARY OF IMPLICATIONS OF BUILD STRATEGY ON COST AND LEAD TIME

by increasing the number of furnaces or operators on the shopfloor, the system can decrease both the lead and total production times. Presumably, by utilizing more AM machines yet keeping the same number of ovens or workers, the production system experiences a bottleneck at the heat treatment step. This effect is demonstrated by the inversion points of the black and blue curves (Fig. 6, bottom right) indicating the throughput blockages in the configurations with only one furnace and more than three AM machines.

\section{CONCLUSION}

The design of a cost- and time-efficient AM production facility plays a critical role in supporting industrial confidence in the adoption of AM technologies. From the current study, we demonstrate the complexity of optimizing resources expenditure through the configuration of production assets and the selection of production strategies, at both the system and individual object levels. A preliminary simulation experiment which compares four aspects of these planning decisions - the number of printers, furnaces, operators, and the batching strategy - already yields a wide range of performance variations. For production of a single part type, the single strategy can reduce the lead time by a factor of 2 but increase the cost per part by a factor of 1.5. This cost is driven by greater expenditure for powder and time for auxiliary and waiting processes. By utilizing additional machines, the lead time per part can also be improved, yet with a significantly lower cost premium at high production volumes. For production of multiple part types, going from either max fill or mixed max fill strategies to the single strategy reduces the lead times and build preparation costs, but lowers the overall productivity and increases all other costs constituents significantly, most notably the cost of the printing operation itself. Presumably, the costefficient application of the single strategy would require the dedicated "high priority" machines, or deployment exclusively when the factory is at low overall utilization.

There are, however, several shortcomings associated with the model. First, the model should be capable of analyzing a broader set of scenarios (e.g. to enable comparison of different machine architectures, to account for parts composed of different materials, and so on). Second, a consideration of inspection operations should be given, and the associated costs need to be included. Given strong industrial interest in the qualification and inspection of AM-produced components, this must be considered as an essential task (and therefore cost and time element) in any 
production scenario. Third, the model uses simplistic terms when calculating certain operations - e.g., volumetric constants are used for estimating build time or gas consumption. Integration with external simulations or estimators would enable greater accuracy and configurability and is the subject of future work. Finally, the model needs refinement through the validation with real case study data.

\section{ACKNOWLEDGEMENTS}

This work was supported by funding from the MIT Center for Additive and Digital Advanced Production Technologies (APT), the National Science Foundation Graduate Research Fellowship Program (to K.G.), the Ford-MIT Alliance, and the Skolkovo Institute of Science and Technology's Center for Design, Manufacturing and Materials.

$\begin{array}{ccc}\text { - Mixed max fill } & \boldsymbol{\Delta} \text { Single } & \bullet \text { Max fill } \\ - & -1 \text { operator } / 1 \text { furnace }-1 \text { operator } / 5 \text { furnaces }-3 \text { operators } / 3 \text { furnaces }-5 \text { operators } / 1 \text { furnace }-5 \text { operators } / 5 \text { furnaces }\end{array}$
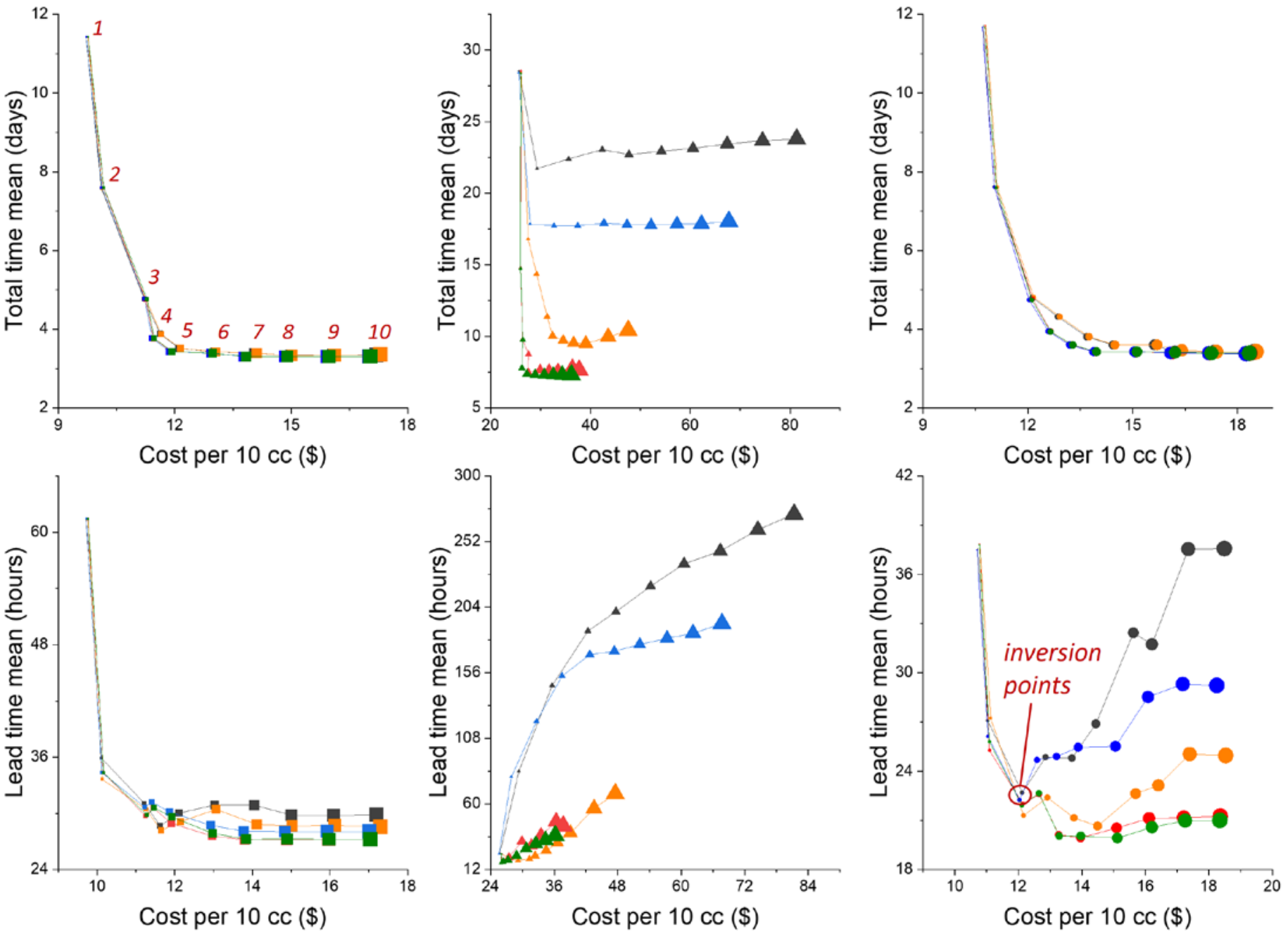

FIGURE 6: COMPARISON OF AVERAGE COST METRIC (\$CC) VERSUS AVERAGE LEAD TIME AND TOTAL TIME OF PRODUCTION. THE SYMBOL SIZE DESIGNATES AN INTEGER VALUE OF PRINTERS UTILIZED: FROM THE SMALLEST FOR ONE MACHINE TO THE LARGEST FOR TEN MACHINES

\section{REFERENCES}

[1] M. Schmidt et al., "Laser based additive manufacturing in industry and academia," CIRP Annals - Manufacturing Technology, 2017.
[2] O. Andersson et al., "Developing additive manufacturing technology for burner repair," Proceedings of the ASME Turbo Expo, 2016. 
[3] M. Schneck et al., "Evaluating the use of additive manufacturing in industry applications", Procedia CIRP - 52nd CIRP Conference on Manufacturing Systems, vol. 81, pp. 19-23, 2017.

[4] T. Wohlers et al., " $3 \mathrm{D}$ Printing and additive manufacturing state of the industry : annual worldwide progress report”, Wohlers Associates, Inc, 2019.

[5] ISO (International Organization for Standardization)/TC ASTM (American Society for Testing and Materials), Standard terminology for additive manufacturing - General principles Terminology. ISO/TC 261-ASTM F42.

[6] W.E. Frazier, "Metal Additive Manufacturing: A Review," Journal of Materials Engineering and Performance, vol. 23, no. 6, pp. 1917-1928, 2014.

[7] N. Garzaniti, A. Golkar and P. Maggiore, "Additive Manufacturing Evaluation Tool for Design Studies”, IEEE Systems Journal (Early Access), 2019.

[8] A. Niazi et al., "Product Cost Estimation: Technique Classification and Methodology Review," ASME. J. Manuf. Sci. Eng., vol. 128, no. 2, pp. 563-575, 2006.

[9] M. Jahangirian et al., "Simulation in manufacturing and business: A review," European Journal of Operational Research, vol. 203, no. 1, pp. 1-13, 2010.

[10] S. Mounsey, B. Hon and C. Sutcliffe, "Performance modelling and simulation of metal powder bed fusion production system,” CIRP Annals, vol. 65, no. 1, pp. 421-424, 2016. 